\title{
USO DE RECURSOS Y COSTOS ASOCIADOS A LAS FRACTURAS EN MUJERES ESPAÑOLAS
}

\author{
Antoni Sicras-Mainar ${ }^{1, a}$, Ruth Navarro-Artieda ${ }^{2, b}$, Jordi Ibáñez-Nolla 3 ,c
}

\begin{abstract}
RESUMEN
El objetivo del estudio fue determinar el uso de recursos y costos debidos a fracturas óseas en mujeres españolas mayores de 50 años en un ámbito poblacional. Se realizó un estudio observacional y retrospectivo, en seis centros de atención primaria y dos hospitales urbanos de España. Se registró datos sociodemográficos, de comorbilidad, uso de recursos (consultas médicas en atención primaria, pruebas complementarias, medicación, atención especializada, hospitalizaciones, visitas, urgencias), costos y pérdida de productividad. Se incluyeron los registros de 19022 mujeres de los cuales el $7 \%$ presentó algún tipo de fractura entre el 2003 y 2007. Las fracturas se asociaron mayoritariamente con osteoporosis (OR: 3,2 ), fibromialgia (OR: 2,4 ) y alteraciones tiroideas (OR: 2,2). En el modelo corregido, el costo total para las pacientes que tuvieron fractura fue de USD 3727 mientras que en las que no la tuvieron fue USD $2705,5$ ( $p<0,001)$. Las pacientes con fractura generan un mayor uso de recursos, costos sanitarios, y pérdidas de productividad laboral.
\end{abstract}

Palabras clave: Fracturas óseas; Osteoporosis; Recursos en Salud; Costos y análisis de costo (fuente: DeCS BIREME).

\section{USE OF RESOURCES AND COSTS ASSOCIATED TO FRACTURES IN SPANISH WOMEN}

\begin{abstract}
The objective of the study was to determine the use of resources and costs due to bone fractures in Spanish women above 50 years of age in the population scope. An observational and retrospective study was conducted in six primary care centers and two urban hospitals in Spain. Socio-demographic and co-morbidity data, use of resources (primary care consultations, complementary tests, medications, specialized care, hospitalizations, visits, urgencies), costs and productivity losses were registered. Records of 19022 women were included, $7 \%$ showed some type of fracture between 2003 and 2007. Fractures were mostly associated with osteoporosis (OR: 3.2), fibromyalgia (OR: 2.4) and thyroid changes (OR: 2.2). In the corrected model, the total cost for patients who had a fracture was USD 3727 compared to USD $2705.5(p<0.001)$ for those who did not have it. Patients with a fracture generate a greater use of resources, sanitation costs and work productivity losses.
\end{abstract}

Key words: Fractures, bone; Osteoporosis; Health resources; Costs and cost analysis (source: MeSH NLM).

\section{INTRODUCCIÓN}

La osteoporosis (OP) es una enfermedad caracterizada por la disminución de la masa ósea y el deterioro de la microarquitectura del tejido óseo ${ }^{(1-4)}$. En los países industrializados, la OP es considerada un problema de salud pública de gran magnitud y como la enfermedad metabólica ósea más prevalente, ya que afecta al $35 \%$ de las mujeres mayores de 50 años, mientras que para edades superiores a 70 años, el porcentaje se eleva a $52 \%{ }^{(3-8)}$. No obstante, la prevalencia de la OP puede plantear dificultades por la definición que se utilice; por los rangos de densidad mineral ósea que se establezcan para describir la población normal; por las técnicas de medida usadas, o por el subdiagnóstico de los casos. Debido a ello, algunos estudios epidemiológicos valoran la OP a través de la evaluación de su manifestación clínica

\footnotetext{
Dirección de Planificación, Badalona Serveis Assistencials SA. Barcelona, España.

Documentación Médica, Hospital Germans Trias i Pujol. Barcelona, España.

Dirección Asistencial, Badalona Serveis Assistencials SA. Barcelona, España.

Médico de familia, doctor en Medicina; ${ }^{\mathrm{b}}$ médico especialista en documentación médica; ${ }^{\mathrm{c}}$ médico internista

Recibido: 13-01-12 Aprobado: 25-07-12
}

Citar como: Sicras-Mainar A, Navarro-Artieda R, Ibáñez-Nolla J. Uso de recursos y costos asociados a las fracturas en mujeres españolas. Rev Peru Med Exp Salud Publica. 2012;29(3):350-6. 
principal: las fracturas osteoporóticas (FO), problema estrechamente asociado con la enfermedad.

Estas fracturas se relacionan con tres localizaciones principales: cadera, columna vertebral y muñeca $(1,3,4,9-11)$. Los estudios realizados constatan grandes variaciones en la incidencia y prevalencia de FO entre diferentes países, regiones, grupos poblacionales y medio residencial ${ }^{(1,3,4)}$. El hecho de que gran parte de las fracturas vertebrales sean asintomáticas podría explicar que algunos trabajos señalen cifras superiores comparadas con los estudios sobre fractura de cadera, mientras que otros indican cifras similares o incluso inferiores. Otros estudios realizados en España, en mujeres de más de 65 años, mostraron una incidencia global de fractura de muñeca de más del doble en relación a la de cadera, y una incidencia similar a la de fractura de húmero ${ }^{(12-16)}$.

La OP tiene un gran impacto sobre el estado de salud de la población, y supone una gran sobrecarga económica para el sistema sanitario ${ }^{(17)}$. Sin embargo, la evidencia disponible sobre el impacto económico de las fracturas, ha sido mayoritariamente obtenida de modelos experimentales de evaluación económica ${ }^{(18-20)}$. El objetivo de este trabajo fue determinar el uso de recursos y costos (tanto sanitarios como pérdida de productividad laboral) posteriores a una fractura, en mujeres españolas de edad superior a 50 años (potencialmente postmenopáusicas) en un ámbito poblacional.

\section{EL ESTUDIO}

El presente trabajo es un estudio observacional de carácter retrospectivo. Se basó en la revisión de los registros médicos (bases de datos informatizadas) de pacientes seguidas en régimen ambulatorio y hospitalario, provenientes de un sector urbano-industrial y de nivel socioeconómico medio-bajo. Las pacientes habían sido atendidas en seis centros españoles de atención primaria (AP), en el Hospital Municipal de Badalona o en el Hospital Universitario Germans Trías y Pujol.

Se incluyeron los registros de mujeres mayores de 50 años que demandaron atención médica y presentaron algún tipo de fractura ósea entre los años 2003 y 2007. Se excluyó la información de las pacientes con antecedentes de neoplasia y de enfermedades metabólicas óseas (raquitismo u osteomalacia, hiperparatiroidismo y enfermedad ósea de Paget). Se utilizó como grupo control una cohorte formada por mujeres mayores de 50 años atendidas durante el mismo periodo, con iguales criterios de exclusión, y sin antecedentes de fracturas óseas.
El diagnóstico de fractura ósea se obtuvo a partir de la Clasificación Internacional de la AP (CIAP-2), en el componente 7 de las enfermedades y problemas de salud (21): fractura de cúbito/radio (L72); fractura de tibia/peroné (L73); fractura de los huesos de la mano/ pie (L74); fractura de fémur/cadera (L75); fractura vertebral (L76.1); fractura costal (L76.2); fractura de húmero (L76.3), u otros tipos de fracturas (L76.9). Las principales variables de estudio fueron: edad (continua y por rangos) y antecedentes personales obtenidos a partir de la CIAP-2 (21): hipertensión arterial (K86, K87); diabetes mellitus (T89,T90); dislipemia (T93); obesidad (T82); tabaquismo (P17); alcoholismo (P15, P16); todos los tipos de fallos orgánicos (cardiaco, hepático y renal); cardiopatía isquémica (K74, K76, K75); accidente vásculocerebral (K90, K91, K93); enfermedad pulmonar obstructiva crónica (R95); asma bronquial (R96), y demencias o trastornos de memoria (P70, P20); enfermedades neurológicas: enfermedad de Parkinson (N87); epilepsia (N88); esclerosis múltiple (N86) y otras enfermedades neurológicas (N99); síndrome depresivo (P76); fibromialgia (L18); alteraciones tiroideas (todos los tipos) y OP (L95). Como variable resumen de la comorbilidad general, para cada paciente atendida, se utilizó el índice de comorbilidad de Charlson (aproximación a la gravedad) ${ }^{(22)}$ y el promedio de episodios por paciente. Se utilizó la tasa de cambio 1 EUR = 1,31050 USD.

Los recursos a recoger en el estudio se definieron teniendo en cuenta las características de la organización y el grado de desarrollo de los sistemas de información disponibles. Se consideraron costos directos sanitarios (costos directos, perspectiva del financiador); los relacionados con la actividad asistencial de los profesionales (visitas médicas, días de hospitalización, urgencias, solicitudes diagnósticas o terapéuticas y medicación dispensada), y se consideraron costos no sanitarios (costos indirectos), los relativos a la pérdida de productividad laboral (número de bajas y días de incapacidad laboral). La unidad de producto que sirvió de base al cálculo final (durante el periodo de estudio) fue el paciente atendido, y el costo fue expresado por paciente (costo unitario). Los diferentes conceptos de estudio y su valoración económica se detallan en la Tabla 1 (correspondientes al año 2006). Las diferentes tarifas se obtuvieron a partir de la contabilidad analítica de los centros, excepto para la medicación y para los días de baja laboral. El costo de la medicación se calculó a partir de las prescripciones (recetas médicas agudas, crónicas o a demanda) tomando el precio de venta al público por envase en el momento de la prescripción y para el costo de los días de baja laboral se utilizó el salario mínimo interprofesional (fuente: Instituto Nacional de Estadística). Para las pacientes 
Tabla 1. Costos unitarios de los recursos sanitarios y de las pérdidas de productividad laboral, año 2006.

\begin{tabular}{lc}
\hline Recursos sanitarios y no sanitarios & $\begin{array}{c}\text { Costos unitarios } \\
\text { USD }\end{array}$ \\
\hline Visitas médicas & \\
\hline Visita médica a atención primaria & 28,6 \\
\hline Visita médica a urgencias & 145,1 \\
\hline Hospitalización (un día) & 396,3 \\
$\quad$ Visita médica a atención & 128,9 \\
$\quad$ especializada & \\
Pruebas complementarias & 27,5 \\
$\quad$ Pruebas de laboratorio & 22,8 \\
\hline Radiología convencional & 45,9 \\
\hline Pruebas diagnósticas/terapéuticas & \\
Productividad laboral & 68,8 \\
\hline Costo por día no trabajado &
\end{tabular}

Fuente de los recursos sanitarios: contabilidad analítica propia.

sin fractura se contabilizaron los recursos utilizados durante el año 2006. El seguimiento de estos pacientes fue de un año.

El estudio fue aprobado por el Comité de Ética de Investigación Clínica del Hospital Universitario Germans
Trías y Pujol de Badalona (Barcelona, España). Se efectuó un análisis estadístico descriptivo, univariado con valores de media, desviación estándar (DE) e intervalos de confianza (IC) del 95\%. En el análisis bivariado se utilizaron las pruebas de t de Student y chi cuadrado. Se efectuó un análisis de regresión logística para corregir las variables asociadas a la presencia de fractura (variable dependiente). La comparación del costo total (ambulatorio y hospitalario) se realizó según las recomendaciones de Thompson y Barber (23), quienes sugieren el uso de pruebas paramétricas a pesar de seguir una distribución no normal. Se realizó análisis de la covarianza (ANCOVA), con la edad y el índice de Charlson como covariables (estimación de medias marginales con ajuste de Bonferroni). Se utilizó el programa SPSS versión 17, estableciendo una significación estadística para valores de $p<0,05$.

Para el período de estudio se incluyeron los registros de 19022 mujeres que cumplían con los criterios de inclusión. La edad media fue de 65,8 años, el promedio del índice de Charlson fue de 0,4 y el promedio de episodios/año de 6,8 . Las fracturas más frecuentes fueron las de cúbito/radio

Tabla 2. Características generales de los pacientes según presencia o ausencia de fractura ósea.

\begin{tabular}{|c|c|c|c|c|}
\hline & \multicolumn{4}{|c|}{ Grupos de estudio } \\
\hline & $\begin{array}{c}\text { Sin fracturas } \\
n=17685\end{array}$ & $\begin{array}{c}\text { Con fracturas } \\
n=1337\end{array}$ & $\begin{array}{c}\text { Total } \\
n=19022\end{array}$ & p \\
\hline \multicolumn{5}{|l|}{ Datos sociodemográficos } \\
\hline Edad & $65,4(11,2)$ & $70,9(11,5)$ & $65,8(11,3)$ & $<0,001$ \\
\hline \multicolumn{5}{|l|}{ Comorbilidad general } \\
\hline Promedio de episodios & $6,5(3,9)$ & $7,9(4,3)$ & $6,8(3,9)$ & $<0,001$ \\
\hline Índice de Charlson & $0,4(0,6)$ & $0,5(0,7)$ & $0,4(0,7)$ & $<0,001$ \\
\hline \multicolumn{5}{|l|}{ Comorbilidad asociada } \\
\hline Hipertensión arterial & $42,7 \%$ & $52,0 \%$ & $43,4 \%$ & $<0,001$ \\
\hline Diabetes mellitus & $14,7 \%$ & $20,0 \%$ & $15,0 \%$ & $<0,001$ \\
\hline Dislipemia & $41,2 \%$ & $44,8 \%$ & $41,4 \%$ & 0,008 \\
\hline Obesidad & $19,8 \%$ & $22,3 \%$ & $20,0 \%$ & 0,025 \\
\hline Fumadores activos & $10,5 \%$ & $5,7 \%$ & $10,1 \%$ & $<0,001$ \\
\hline Alcoholismo & $0,6 \%$ & $1,3 \%$ & $0,6 \%$ & 0,002 \\
\hline Cardiopatía isquémica & $4,1 \%$ & $6,7 \%$ & $4,3 \%$ & $<0,001$ \\
\hline ACV & $6,2 \%$ & $10,6 \%$ & $6,5 \%$ & $<0,001$ \\
\hline Eventos cardiovasculares & $9,5 \%$ & $15,8 \%$ & $9,9 \%$ & $<0,001$ \\
\hline Insuficiencias orgánicas & $9,3 \%$ & $13,4 \%$ & $9,6 \%$ & $<0,001$ \\
\hline Asma bronquial & $5,7 \%$ & $7,0 \%$ & $5,8 \%$ & NS \\
\hline EPOC & $1,7 \%$ & $3,2 \%$ & $1,8 \%$ & $<0,001$ \\
\hline Neuropatías & $0,9 \%$ & $1,5 \%$ & $1,0 \%$ & 0,045 \\
\hline Demencias & $2,3 \%$ & $6,3 \%$ & $2,5 \%$ & $<0,001$ \\
\hline Síndrome depresivo & $24,3 \%$ & $30,0 \%$ & $24,7 \%$ & $<0,001$ \\
\hline Fibromialgia & $8,6 \%$ & $20,1 \%$ & $9,4 \%$ & $<0,001$ \\
\hline Alteraciones tiroideas & $8,1 \%$ & $19,4 \%$ & $9,0 \%$ & $<0,001$ \\
\hline Osteoporosis ${ }^{a}$ & $32,4 \%$ & $48,5 \%$ & $34,2 \%$ & $<0,001$ \\
\hline
\end{tabular}

ACV: accidente cerebro vascular, EPOC: enfermedad pulmonar obstructiva crónica.

Valores expresados en media (DE: desviación estándar) o porcentaje; p: significación estadística; NS: no significativo.

${ }^{a}$ Diagnóstico de OP referido al momento de realizar el análisis. 
Tabla 3. Uso de recursos sanitarios y no sanitarios según la ausencia o presencia de fractura ósea.

\begin{tabular}{lcccc}
\hline & \multicolumn{3}{c}{ Grupos de estudio } \\
\cline { 2 - 5 } & $\begin{array}{c}\text { Sin fracturas } \\
\mathbf{n = 1 7 6 8 5}\end{array}$ & $\begin{array}{c}\text { Con fracturas } \\
\mathbf{n = 1 3 3 7}\end{array}$ & $\begin{array}{c}\text { Total } \\
\mathbf{n = 1 9 0 2 2}\end{array}$ & $\mathbf{p}$ \\
\hline Recursos sanitarios & & & & \\
\hline Visitas médicas (atención primaria) & $11,7 \pm 10,7$ & $16,0 \pm 13,0$ & $12,1 \pm 10,0$ & $<0,001$ \\
\hline Pruebas de laboratorio & $4,9 \pm 3,9$ & $6,4 \pm 4,4$ & $5,0 \pm 3,9$ & $<0,001$ \\
\hline Radiología convencional & $2,1 \pm 2,5$ & $2,8 \pm 2,7$ & $2,2 \pm 2,5$ & $<0,001$ \\
\hline Pruebas complementarias & $0,1 \pm 0,4$ & $0,3 \pm 0,7$ & $0,1 \pm 0,6$ & $<0,001$ \\
\hline Visitas médicas (especializada) & $2,5 \pm 3,6$ & $4,4 \pm 3,7$ & $2,6 \pm 3,6$ & $<0,001$ \\
\hline Días de hospitalización & $0,4 \pm 2,7$ & $1,0 \pm 5,1$ & $0,5 \pm 3,0$ & $<0,001$ \\
\hline Consultas a urgencias & $0,4 \pm 0,8$ & $0,7 \pm 1,1$ & $0,4 \pm 0,9$ & $<0,001$ \\
\hline Recursos no sanitarios & & & $10,3 \pm 45,0$ & $\mathrm{NS}$ \\
\hline Días de incapacidad laboral & $10,2 \pm 45,2$ & $11,0 \pm 43,2$ & & \\
\hline
\end{tabular}

Valores expresados en media (DE: desviación estándar); p: significación estadística; NS: no significativo.

$(23,3 \%)$ y las de la mano/pie (21,3\%). En la corrección del modelo logístico, las pacientes con fractura se asociaron con OP (OR: 3,2), fibromialgia (OR: 2,4), alteraciones tiroideas (OR: 2,2) y demencia (OR: 1,7), p<0,05 en todos los casos. En la Tabla 2 se muestran las características sociodemográficas y las comorbilidades de las pacientes.

La Tabla 3 muestra los recursos anuales utilizados según la presencia o no de fractura. Globalmente (considerando conjuntamente la población con y sin fractura) los costos totales desagregados en sanitarios (directos) y pérdidas de productividad laboral (no sanitarios, indirectos) fueron del 74,5 y
25,5\%, respectivamente (costo promedio/unitario: USD 2070 y USD 706; p<0,001). En la Tabla 4 se muestran los costos no corregidos de los dos grupos estudiados. La Tabla 5 señala el modelo de costos corregidos.

\section{DISCUSIÓN}

El presente estudio pretende determinar el uso de recursos y costos (sanitarios y pérdida de productividad laboral) debidos a las fracturas óseas en mujeres españolas de edad superior a 50 años (potencialmente

Tabla 4. Modelo no corregido de costos sanitarios y no sanitarios según la ausencia o presencia de fractura ósea.

\begin{tabular}{|c|c|c|c|c|}
\hline & \multicolumn{4}{|c|}{ Grupos de estudio } \\
\hline & $\begin{array}{c}\text { Sin fracturas } \\
n=17685\end{array}$ & $\begin{array}{c}\text { Con fracturas } \\
n=1337\end{array}$ & $\begin{array}{c}\text { Total } \\
n=19022\end{array}$ & p \\
\hline & USD & USD & USD & \\
\hline Costos sanitarios totales & $1984,3 \pm 1627,1$ & $3204,0 \pm 2373,7$ & $2069,9 \pm 1707,0$ & $<0,001$ \\
\hline Costos en atención primaria & $1436,9 \pm 1037,1$ & $2125,7 \pm 1252,4$ & $1485,3 \pm 1062,2$ & $<0,001$ \\
\hline Visitas médicas & $651,6 \pm 363,4$ & $710,7 \pm 439,9$ & $534,0 \pm 371,1$ & $<0,001$ \\
\hline Pruebas de laboratorio & $135,7 \pm 81,5$ & $176,7 \pm 92,8$ & $138,6 \pm 82,3$ & $<0,001$ \\
\hline Radiología convencional & $48,7 \pm 43,9$ & $63,1 \pm 46,3$ & $49,9 \pm 44,2$ & $<0,001$ \\
\hline Pruebas complementarias & $6,2 \pm 15,5$ & $12,4 \pm 24,5$ & $6,7 \pm 16,4$ & $<0,001$ \\
\hline Prescripción farmacéutica & $725,7 \pm 757,7$ & $1162,8 \pm 960,9$ & $756,4 \pm 778,4$ & $<0,001$ \\
\hline Costos en atención especializada & $547,3 \pm 1002,1$ & $1078,3 \pm 1723,5$ & $584,7 \pm 1073,8$ & $<0,001$ \\
\hline Visitas médicas & $321,6 \pm 352,4$ & $568,8 \pm 366,2$ & $339,0 \pm 356,6$ & $<0,001$ \\
\hline Hospitalización & $168,6 \pm 829,1$ & $412,9 \pm 1556,8$ & $185,8 \pm 900,9$ & $<0,001$ \\
\hline Urgencias & $57,1 \pm 93,8$ & $96,5 \pm 126,4$ & $60,0 \pm 96,7$ & $<0,001$ \\
\hline Costos no sanitarios (productividad) & $703,6 \pm 2373,4$ & $757,6 \pm 2271,6$ & $707,3 \pm 2366,4$ & NS \\
\hline Costos totales & $2687,7 \pm 2914,6$ & $3961,6 \pm 3231,4$ & $2777,3 \pm 2948,4$ & $<0,001$ \\
\hline
\end{tabular}

Valores expresados en media (DE: desviación estándar); p: significación estadística; NS: no significativo. 
Tabla 5. Modelo de costos corregidos sanitarios y no sanitarios según la ausencia o presencia de fractura ósea.

\begin{tabular}{|c|c|c|c|}
\hline & $\begin{array}{c}\text { Sin fracturas } \\
n=17685\end{array}$ & $\begin{array}{c}\text { Con fracturas } \\
n=1337\end{array}$ & $p^{a}$ \\
\hline & USD (IC95\%) & USD (IC95\%) & \\
\hline $\begin{array}{l}\text { Costos } \\
\text { sanitarios totales }\end{array}$ & $\begin{array}{c}2018,1 \\
(1988,6-2760,2)\end{array}$ & $\begin{array}{c}2757,2 \\
(2650,1-2863,7)\end{array}$ & $<0,001$ \\
\hline $\begin{array}{l}\text { - Costos en } \\
\text { atención } \\
\text { primaria }\end{array}$ & $\begin{array}{c}1459,7 \\
(1441,0-1477,7)\end{array}$ & $\begin{array}{c}1822,3 \\
(1758,1-1887,7)\end{array}$ & $<0,001$ \\
\hline $\begin{array}{l}\text { - Costos en } \\
\text { atención } \\
\text { especializada }\end{array}$ & $\begin{array}{c}558,2 \\
(538,4-1164,6)\end{array}$ & $\begin{array}{c}934,8 \\
(860,7-1008,7)\end{array}$ & $<0,001$ \\
\hline $\begin{array}{l}\text { Costos no } \\
\text { sanitarios }\end{array}$ & $\begin{array}{c}687,5 \\
(641,9-731,1)\end{array}$ & $\begin{array}{c}969,4 \\
(805,3-1133,5)\end{array}$ & 0,001 \\
\hline Costos totales & $\begin{array}{c}2705,5 \\
(2650,1-2760,2)\end{array}$ & $\begin{array}{c}3726,6 \\
(3525,1-3928,1)\end{array}$ & $<0,001$ \\
\hline
\end{tabular}

Valores expresados en media; p: significación estadística; IC: intervalo de confianza. ${ }^{\text {a }}$ Modelo de ANCOVA.

postmenopáusicas), en comparación con un grupo control formado por una población de referencia de características similares pero sin fractura. Si bien el mayor porcentaje del costo se genera durante el primer año de seguimiento, este no se limita almencionado periodo. Cabe destacar que, sin una adecuada estandarización de las metodologías, los resultados obtenidos deben ser interpretados con prudencia, y dentro del escenario de proveedor de servicios propio. Ello nos obliga a ser cautelosos en la valoración de la validez externa de dichos resultados ${ }^{(24)}$.

El estudio mostró que el $34,2 \%$ de las mujeres de edad superior a 50 años tenían OP y que el $7 \%$ tuvo algún tipo de fractura. Los valores de prevalencia de la OP concuerdan con la mayoría de los estudios revisados ${ }^{(1,3,4,9,10)}$. No obstante, en cuanto al riesgo de padecer una fractura y su localización anatómica, la comparación de los resultados ofrece mayor dificultad, en función del ámbito de realización y la metodología del trabajo. En este aspecto, algunos autores sugieren que casi la mitad de las mujeres sufrirán alguna FO antes de llegar a los 70 años ${ }^{(3,8)}$. En general, 20\% sufrirá una fractura de muñeca, $25 \%$ una fractura vertebral y la cantidad de mujeres con fractura de cadera se irá incrementando, por el envejecimiento progresivo de la población (9,11). El porcentaje de fractura más bajo, en la serie estudiada, se observó en la fractura vertebral. Esta circunstancia podría explicarse por el hecho de que gran parte de estas son asintomáticas, o tal vez por un efecto de subregistro. Un estudio realizado en España en mujeres de más de 65 años, mostró una incidencia global de fractura de muñeca mayor al doble en relación con la de cadera, y una incidencia similar a la de fractura de húmero ${ }^{(15)}$. Nuestro estudio está más relacionado con este tipo de resultados de base poblacional ${ }^{(1,3,9,25,26)}$.

Es conocido que no todos los pacientes experimentan un restablecimiento completo después de la fractura, ya que alrededor del $35 \%$ de estos enfermos sufren síntomas persistentes. Además, el costo de las $\mathrm{FO}$ va mucho más allá del propio tratamiento, cirugía en cada caso, rehabilitación y calidad de vida del paciente. La fractura de cadera produce una discapacidad que implica la necesidad de asistencia, dado que un gran porcentaje de estas personas no pueden efectuar sus labores cotidianas. La fractura vertebral se relaciona con presencia de deformaciones hasta en un $25 \%$ de los casos, mientras que la fractura de muñeca presenta una discapacidad mucho más reducida, con solo un $2 \%$ de los pacientes que tendrían alguna dependencia residual ${ }^{(27)}$.

El costo total (sanitario/no sanitario) promedio por paciente fue mayor en las pacientes que presentaron una fractura con relación al grupo de referencia, siendo los costos más importantes los utilizados en AP. En un estudio de casos y controles efectuado en los Estados Unidos, el valor medio de los costos médicos fue de USD 761 y 625 para los casos y controles, y USD 3884 y 712, respectivamente, al cabo de un año después de la fractura. El mayor costo fue para la fractura de cadera (USD 11241), seguido de las vertebrales y de las de muñeca (USD 1955 y 1628, respectivamente) ${ }^{(28)}$. Desai et al., al comparar los costos totales de la atención en mujeres con OP y fractura, observó que el $68 \%$ de las pacientes en los grupos de fracturas estaba recibiendo terapia con medicamentos para su prevención, y que el mayor porcentaje del costo se concentró en los servicios ambulatorios (38\%), seguido por servicios de hospitalización (24\%) y farmacia $(21 \%)^{(29)}$. Ante la falta de estudios comparativos y con las limitaciones metodológicas en cuanto a la posibilidad de comparación, nuestros resultados ofrecen una cierta similitud porcentual con los de estos estudios.

Las posibles limitaciones del presente estudio se relacionan con la categorización de la enfermedad, el posible sesgo de clasificación de las pacientes y el método de cálculo de los costos, atribuibles al sistema de información desarrollado. Asimismo, al ser un estudio con diseño observacional retrospectivo, también se ve afectado por el subregistro. Otra posible limitación del estudio hace referencia a las fracturas expuestas, que requieren un tratamiento diferente por cirugía reconstructiva u otro tipo de rehabilitación; por lo que podría asumirse un costo diferente. Además, no existe un único protocolo de actuación, por lo que el cálculo de los costos está sujeto a la variabilidad de la práctica 
clínica. Al no haber actualizado las tarifas del estudio al periodo de reclutamiento de los pacientes, los costos obtenidos pueden sufrir cierto grado de incertidumbre. Cabe destacar que se utilizó una fuente secundaria de información, por lo que las pacientes no fueron interrogadas o evaluadas directamente. El presente estudio se ha realizado en un área geográfica limitada $\mathrm{y}$, en consecuencia, difícilmente generalizable. Por ello, sería interesante reproducir el estudio en otras organizaciones o áreas sanitarias.

El éxito de la atención a los pacientes con enfermedades crónicas, como la FO debería basarse en intervenciones de equipos pluridisciplinarios, que promuevan intervenciones efectivas, en las que los pacientes estén altamente comprometidos en su autocuidado. En conclusión, las pacientes con fracturas generan un mayor costo sanitario y pérdida de productividad laboral en comparación con una cohorte similar sin fracturas. Además, es importante destacar que casi la mitad de las pacientes que sufrieron una fractura tenían un diagnóstico previo establecido de OP.

Contribuciones de autoría: ASM y RNA participaron en la concepción y diseño del trabajo, análisis e interpretación de datos, y redacción de la primera versión del manuscrito, JIN participó en la interpretación de datos, y redacción de la primera versión del manuscrito. Todos los autores revisaron en forma crítica versiones preliminares del manuscrito y aprobaron la versión final del trabajo.

Fuentes de financiamiento: autofinanciado.

Conflictos de interés: los autores declaran no tener conflictos de interés en la publicación de este artículo.

\section{REFERENCIAS BIBLIOGRÁFICAS}

1. NIH Consensus Development Panel on Osteoporosis Prevention, Diagnosis, and Therapy. Osteoporosis prevention, diagnosis, and therapy. JAMA. 2001;285(6):785-95.

2. Muñoz-Torres M, Alonso G, MezquitaRaya P. Prevención y tratamiento de la osteoporosis. Endocrinol Nutr. 2003;50:1-7.

3. World Health Organization (WHO). Assessments of fracture risk and its application to screening for postmenopausal osteoporosis. Report of a WHO Study Group. Geneva: WHO; 1994.

4. Grupo de Trabajo de la Sociedad Española de Investigaciones Oseas y Metabolismo Mineral. Osteoporosis posmenopáusica. Guía de práctica clínica. Rev Clin Esp. 2003;203(10):496-506.

5. Johnell O, Kanis JA. An estimate of the worldwide prevalence and disability associated with osteoporotic fractures. Osteoporos Int. 2006;17(12):1726-33.

6. Lespessailles E, Cotté FE, Roux C, Fardellone P, Mercier F, Gaudin AF. Prevalence and features of osteoporosis in the French general population: the Instant study. Joint Bone Spine. 2009;76(4):394-400.

7. Cheng H, Gary LC, Curtis JR, Saag KG, Kilgore ML, Morrisey MA, et al. Estimated prevalence and patterns of presumed osteoporosis among older Americans based on
Medicare data. Osteoporos Int. 2009;20(9):1507-15.

8. Recker R, Lappe J, Davies KM, Heaney R. Bone remodelling increases substantially in the years after menopause and remains increased in older osteoporosis patients. J Bone Miner Res. 2004;19(10):1628-33.

9. Kanis JA, Johnell O. Requirements for DXA for the management of osteoporosis in Europe. Osteoporos Int. 2005;16(3):229-38.

10. Naves M, Díaz-López JB, Gómez C, Rodríguez-Rebollar A, CannataAndía JB. Determinants of incidence of osteoporotic fractures in the female Spanish population older than 50 . Osteoporos Int. 2005;16(12):2013-7.

11. Sosa M, Saavedra P; Grupo de trabajo en osteoporosis de la Sociedad Española de Medicina Interna (SEMI). Prevalence of vertebral fractures in hip fracture patients. Rev Clin Esp. 2007;207(9):464-8.

12. Panel de Expertos de la Sociedad Española de Reumatología. Documento de la Sociedad Española de Reumatología sobre la osteoporosis posmenopáusica. Rev Esp Reumatol. 2001;28:148-53.

13. Sanders KM, Nicholson GC, Watts JJ, Pasco JA, Henry MJ, Kotowicz MA, et al. Half the burden of fragility fractures in the community occur in women without osteoporosis. When is fracture prevention cost- effective? Bone. 2006;38(5):694700.

14. Vestergaard P, Rejnmark L, Mosekilde L. Increased mortality in patients with a hip fracture-effect of premorbid conditions and post-fracture complications. Osteoporos Int. 2007;18(12):1583-93.

15. Marín F, González-Macías J, Moya R, Onrubia C, Cancelo C, Alvarez $S$, et al. Fractura no vertebral por fragilidad en una cohorte de 5.201 mujeres de 65 o más años durante tres años de seguimiento. Med Clin (Barc). 2006;127:401-4.

16. Lindsay R, Silverman SL, Cooper C, Hanley DA, Barton I, Broy SB, et al. Risk of new vertebral fracture in the year following a fracture. JAMA. 2001;285(3):320-3.

17. Ström O, Borgstrom F, Zethraeus N, Johnell O, Lidgren L, Ponzer S, et al. Long-term cost and effect on quality of life of osteoporosis-related fractures in Sweden. Acta Orthop. 2008;79(2):269-80.

18. Schousboe JT. Cost-effectiveness modeling research of pharmacologic therapy to prevent osteoporosis-related fractures. Curr Rheumatol Rep. 2007;9(1):50-6.

19. Majumdar SR, Lier DA, Beaupre LA, Hanley DA, Maksymowych WP, Juby AG, et al. Osteoporosis case manager for patients with hip fractures: results of a cost-effectiveness analysis conducted alongside a 
randomized trial. Arch Intern Med. 2009;169(1):25-31.

20. Christensen L, Iqbal S, Macarios D, Badamgarav E, Harley C. Cost of fractures commonly associated with osteoporosis in a managed-care population. J Med Econ. 2010;13(2):302-13.

21. Lamberts H, Wood M, Hofmans-Okkes IM, editors. The International Classification of Primary Care in the European Community: with a multi-language layer. Oxford: Oxford University Press; 1993.

22. Charlson ME, Pompei P, Ales KL, MacKenzie CR. A new method of classifying prognostic comorbidity in longitudinal studies: development and validation. J Chronic Dis. 1987;40(5):373-83.

23. Thompson SG, Barber JA. How should cost data in pragmatic randomised trials be analysed?. BMJ. 2000;320(7243):1197-200.
24. Meltzer M. Introduction to Health Economics for Physicians. The Lancet. 2001;358(9286):993-8.

25. Sosa M, Gómez de Tejada MJ. Epidemiología de la fractura vertebral. En: Díaz Curiel M, editor. La fractura vertebral en la práctica clínica. Marid: FHOEMO; 2005. p. 1-6.

26. Briot K, Cortet B, Trémollières F, Sutter B, Thomas T, Roux C, et al. Male osteoporosis: diagnosis and fracture risk evaluation. Joint Bone Spine. 2009;76(2):129-33.

27. Boonen S, Singer AJ. Osteoporosis management: impact of fracture type on cost and quality of life in patients at risk for fracture I. Curr Med Res Opin. 2008;24(6):1781-8.

28. Gabriel SE, Tosteson AN, Leibson CL, Crowson CS, Pond GR, Hammond CS, et al. Direct medical costs attribu- table to osteoporotic fractures. Osteoporos Int 2002;13(4):323-30.

29. Desai SS, Duncan BS, Sloan AS. The cost of treating osteoporosis in a managed health care organization. J Manag Care Pharm. 2003;9(2):142-9.

Correspondencia: Antoni Sicras Mainar Dirección: C. Gaietà Soler, 6-8 entlo. 08911

Badalona. Barcelona, España.

Teléfono: 935072684

Correoelectrónico: asicras@bsa.cat

Consulte las ediciones anteriores de la Revista Peruana de Medicina Experimental y Salud Pública en

\section{www.scielosp.org}

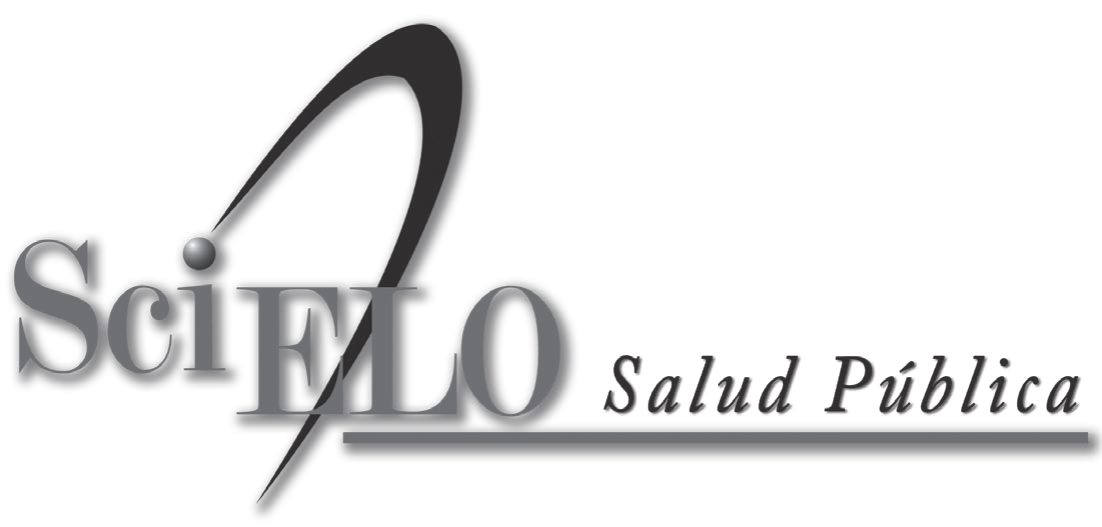

